\section{Adaptación de la lowa Infant Feeding Attitude Scale en población mexicana}

Aguilar-Navarro $\mathrm{HJ}^{1}$, Coronado-Castilleja $\mathrm{A}^{2}$, Gómez-Hernández $\mathrm{OJ}^{2}$, CobosAguilar $\mathrm{H}^{3}$

Resumen

INTRODUCCIÓN: la leche materna es el alimento ideal del neonato. Sin embargo, la frecuencia de la lactancia materna en México ha declinado en la última década hasta 14.4\%. Una manera de enfrentar este problema es identificar las actitudes maternas hacia la lactancia, ya que es un factor modificable. La Escala de Actitudes hacia la Alimentación Infantil de lowa (IIFAS) es utilizada para lograr este objetivo, aunque no se ha validado en México.

OBJETIVO: adaptar la escala de actitudes hacia la alimentación infantil en México.

MATERIALES Y MÉTODOS: estudio transversal con traducción inversa y adaptación cultural, así como consenso entre expertos para la redacción y equivalencia conceptual de los ítems de la escala IIFAS al español, para determinar su validez y fiabilidad.

RESULTADOS: la escala adaptada se aplicó a 385 mujeres grávidas, el $16.3 \%$ mostró actitud positiva hacia la lactancia materna. Se determinó la fiabilidad del Instrumento ( $\alpha$ de Cronbach 0.65 ), así como la validez de constructo con Análisis Factorial Exploratorio.

CONCLUSIONES: la escala de actitudes hacia la alimentación infantil IIFAS versión español (México) puede considerarse válida y confiable para su uso en nuestro medio.

PALABRAS CLAVE: lactancia materna, actitud, validez y confiabilidad.

Acta Pediatr Mex. 2016 May;37(3):149-158.

\section{Adaptation of lowa Infant Feeding Attitude Scale in Mexican population.}

Aguilar-Navarro $\mathrm{HJ}^{1}$, Coronado-Castilleja $\mathrm{A}^{2}$, Gómez-Hernández $\mathrm{OJ}^{2}$, CobosAguilar $\mathrm{H}^{3}$

\section{Abstract}

INTRODUCTION: Breast milk is the ideal food for the infant. However, in Mexico the frequency of breastfeeding has declined in the last decade, to $14.4 \%$. One way to address this problem is to identify maternal attitudes towards breastfeeding, since it is a modifiable factor. The lowa Infant Feeding Attitude Scale (IIFAS) is used to achieve this goal, but has not been validated in Mexico.
${ }^{1}$ Médico Pediatra, coordinador de médicos internos en Pediatría. Vocal de Comité Ética e Investigación de HGZ/MF\#2, IMSS, Monterrey.

${ }^{2}$ Médico Pasante, UDEM.

${ }^{3}$ Coordinación del internado de pregrado, UDEM.

Recibido: 17 de agosto del 2015

Aceptado: 26 de febrero del 2016

Correspondencia

Alejandra Coronado Castilleja

Médico Pasante, UDEM

alecc29@gmail.com

Este artículo debe citarse como Aguilar-Navarro HJ, Coronado-Castilleja A, GómezHernández OJ, Cobos-Aguilar H. Adaptación de la lowa Infant Feeding Attitude Scale en población mexicana. Acta Pediatr Mex 2016;37(3):149-158. 
OBJECTIVE: To adapt scale of attitudes towards infant feeding in Mexico.

MATERIAL AND METHOD: A transversal study with reverse translation and cultural adaptation and consensus among experts for redaction and conceptual equivalence of items of the scale IIFAS to Spanish, to determine their validity and reliability.

RESULTS: The adapted scale was applied to 385 pregnant women, with only $16.3 \%$ showed positive attitude to breastfeeding. Instrument reliability (Cronbach $\alpha 0.65$ ) and construct validity with exploratory factor analysis was determined.

CONCLUSIONS: the scale of attitudes towards infant feeding IIFAS Spanish Mexican version can be considered valid and reliable for use in our environment.

KEYWORDS: breastfeeding; attitude; validity and reliability

\begin{abstract}
${ }^{1}$ Médico Pediatra, coordinador de médicos internos en Pediatría. Vocal de Comité Ética e Investigación de HGZ/MF\#2, IMSS, Monterrey.

${ }^{2}$ Médico Pasante, UDEM.

${ }^{3}$ Coordinación del internado de pregrado,
\end{abstract} UDEM.

\author{
Correspondence \\ Alejandra Coronado Castilleja \\ Médico Pasante, UDEM \\ alecc29@gmail.com
}

\section{INTRODUCCIÓN}

La alimentación en la sociedad actual ha experimentado una transición, ya que de una necesidad biológica, se ha convertido en un fenómeno socio-cultural ${ }^{1}$ y psicológico, ${ }^{2}$ aunado al conocimiento adquirido sobre el tema. ${ }^{3}$ La alimentación durante el primer año de vida e infancia temprana debiera ser la apropiada, por ser la más importante para que un niño se desarrolle y crezca.

En la última década en México, la frecuencia de la alimentación exclusiva al seno materno en menores de seis meses ha declinado. Los resultados de la Encuesta Nacional de Salud y Nutrición (ENSANUT), del año 1999, la ubicaron en 20\%; en el 2006 en $22.3 \%$ y en el 2012 en $14.4 \%{ }^{4}$

A pesar de la amplia gama de beneficios de salud para madres y niños, muchos países aún no han cumplido la recomendación adecuada para una mejor lactancia materna; por ello, varios estudios sugieren que una manera de mitigar este problema sería concentrarse en la lactancia materna identificando los conocimientos y actitudes que presentan la madres, ya que estos factores son modificables. ${ }^{5}$

La indagación de las actitudes a la lactancia materna bajo metodología cuantitativa se realiza a través de diversas escalas. ${ }^{6-10}$ La Escala Infantil de Alimentación y Actitud de lowa (IIFAS) fue diseñada para medir las actitudes maternas de mujeres estadounidenses hacia la alimentación del lactante, con una consistencia alta ( $\alpha$ de 0.86). ${ }^{11}$ La actitud evaluada es un indicador predictivo de la elección del método de alimentación ${ }^{12}$ y en mujeres que amamantan la duración de la lactancia materna. ${ }^{13}$ La escala se ha utilizado en diversos países de habla ingle$\mathrm{sa}^{14,15}$ y se ha traducido y validado a los idiomas rumano, ${ }^{16}$ chino $^{17}$ y japonés. ${ }^{18}$ La escala IIFAS no ha sido validada en México. 


\section{OBJETIVO}

Adaptación de la escala IIFAS en una población del noreste de México.

\section{MÉTODOS}

\section{Diseño}

Se realizó estudio descriptivo transversal para la adaptación cultural de la lowa Infant Feeding Attitude Scale en población mexicana.

\section{Participantes}

Se aplicó la encuesta a 50 participantes para llevar a cabo la prueba piloto, y posteriormente a 385 mujeres en su primera gestación, asistentes a una institución de seguridad social, alfabetizadas y quienes aceptaron participar en el proyecto firmando el consentimiento informado. Se excluyó a mujeres promotoras de lactancia materna o con antecedentes de patologías que contraindicaran la lactancia. El tamaño de la muestra fue mayor de la mínima requerida para las exigencias de los análisis empíricos, ${ }^{19}$ como el análisis estadístico factorial. ${ }^{20,21} \mathrm{El}$ muestreo fue por casos consecutivos.

\section{Instrumento}

La escala original en inglés consta de 17 ítems, de los cuales ocho están redactados de manera favorable a la lactancia materna $3,5,7,9,12,13,15,16$ y el resto hacia la lactancia con fórmula. La escala se evalúa con una graduación de respuesta tipo Likert de cinco puntos, los ítems favorables a lactancia materna, si se elige la opción totalmente en desacuerdo con el ítem, se califica con 1 punto, hasta la opción estar totalmente de acuerdo con el ítem equivale a 5 puntos, la puntuación revierte en los ítems favorables a lactancia con fórmula. ${ }^{11}$
La puntuación total se clasifica en tres categorías: actitud positiva a la lactancia materna, actitud neutral y una actitud positiva a lactancia por fórmula. ${ }^{11}$

Los autores reportan la confiabilidad ( $\alpha$ de Cronbach de 0.86) y la validez con dos estadísticos: con la correlación entre las puntuaciones de la escala y la actitud hacia la alimentación $(r=0.80)$ y con las diferencias entre las puntuaciones de la escala y la planeación de la lactancia (seno materno versus alimentación con fórmula) $(p<0.01){ }^{11}$

Se realizó la adaptación y validación de acuerdo con normas para el desarrollo y validación de instrumentos de medición que se detallan a continuación. ${ }^{22,23}$

\section{Traducción y adaptación cultural al español de México}

Se realizó una traducción inversa: en un primer paso se hizo una traducción directa al español, por dos traductores profesionales con conocimientos médicos. Posteriormente, otros dos traductores tradujeron el instrumento versión español, al idioma inglés. Por último, los cuatro traductores realizaron un consenso de las diferencias, obteniendo finalmente la escala traducida al español.

En la siguiente fase se valoró la escala traducida al español por un comité de cinco expertos en el tema (dos médicos pediatras, un nutriólogo, dos licenciadas en enfermería expertas en tema de lactancia materna) quienes revisaron, en forma ciega e independiente, su redacción y la equivalencia conceptual de los ítems, determinándose un índice de concordancia, obteniendo la primera versión de la escala. Ésta se aplicó en la prueba piloto para evaluar la redacción y obtener un índice de concordancia. 
El comité de expertos evaluó los resultados obteniendo así la segunda versión de la escala, que se retradujo y comparó con la escala original en inglés, realizando cambios mínimos gramaticales para la versión final. La versión final se conformó con los 17 reactivos adaptados transculturalmente, conservando la estructura de las respuestas con la escala de Likert. Una vez terminado lo anterior, se aplicó la versión final de la escala adaptada al español de México a 385 mujeres embarazadas, con un tiempo de resolución de 20 minutos (DE: \pm 6 min), durante el tiempo de espera previo a su consulta de control prenatal o realización de ultrasonido de control en un hospital de seguridad social en Monterrey, durante el año 2014.

\section{Análisis/validación de la escala}

Se utilizó el programa computacional IBM SPSS versión 21 para el análisis de los datos. La fiabilidad se realizó obteniendo la consistencia interna con el estadístico $\alpha$ de Cronbach y los coeficientes ítems-total de cada ítem. Para la validez de constructo se realizó un análisis factorial exploratorio (AFE), por extracción de factores de ejes principales y método de rotación Oblimin para obtener un modelo de agrupación de reactivos que correlacionaron con cada factor. Previo al AFE se aplicó la prueba de Kaiser-Meyer-Olking $(\mathrm{KMO})$ y la prueba de esfericidad de Bartlett.

Para valorar la validez predictiva de la escala se utilizó el constructo: intención de conducta del método de alimentación y los resultados de la actitud obtenida, a través de la prueba correlación de Spearman.

\section{Características sociodemográficas e intención de alimentación}

Se indagó la edad, situación conyugal, el nivel escolar y la vinculación a la actividad económica de acuerdo al marco conceptual ofrecido por el Instituto Nacional de Estadística y Geografía
$(\mathrm{INEGI}),{ }^{24}$ para determinar el nivel socioeconómico de las participantes de acuerdo con la Asociación Mexicana de Agencias de Investigación de Mercado y Opinión Pública (AMAI). ${ }^{25}$ También se identificó el trimestre de la gestación que cursaban las participantes, enfermedades que padecían, su asistencia a pláticas de lactancia materna y por último se preguntó la decisión para la alimentación futura de sus hijos. Se obtuvieron frecuencias de tipos de lactancia elegida, así como del tiempo planeado para alimentar con lactancia materna. ${ }^{26}$

\section{Consideraciones éticas}

El presente proyecto se realizó posteriormente a la autorización por el Comité de Ética e Investigación del hospital, con registro de autorización R-2014-1906-25, guardando el anonimato y confidencialidad de los datos proporcionados por las participantes.

\section{RESULTADOS}

Traducción-adaptación y validez de contenido

Durante el proceso de traducción y adaptación, en la fase de evaluación por el comité de expertos, se obtuvo un índice de Kappa de 0.86 para la concordancia de la equivalencia conceptual de los ítems. Se modificaron los ítems 1, 2, 3, 6,8 y 11 .

Se realizaron pruebas de legibilidad con el índice de Flesch-Szigriszt (52.04). De acuerdo con la Escala Inflesz y al índice de legibilidad, la encuesta es un documento con alguna dificultad para su lectura. ${ }^{27}$

\section{Datos sociodemográficos}

Se aplicó el instrumento a 385 participantes gestantes de su primer embarazo, con un rango de edad entre 14 y 43 años de edad, quienes habitan en 
26 localidades diferentes de cuatro estados de la República Mexicana, el mayor porcentaje fue del municipio de Monterrey, Nuevo León, con 32\% de las participantes. El nivel educativo que predominó fue el nivel medio superior con $40.9 \%$, el $60 \%$ de la población total se clasificó económicamente activa y $64 \%$ casada. El nivel socioeconómico más observado fue la clase media baja con 35\%.

El 33\% de las participantes presentaron enfermedad intercurrente al embarazo, 20\% tuvieron diabetes. Además, 35\% no habían recibido pláticas de orientación de la lactancia y quienes respondieron afirmativamente haberlas recibido, de la totalidad de las participantes, el 23\% recibieron tres pláticas prenatales. Más información de las características sociodemográficas y clínicas de las participantes se muestra en el Cuadro 1.

\section{Puntuaciones de escala IIFAS-versión español México}

La puntuación del instrumento IIFAS-adaptado de las participantes, varió entre 44 y 85 puntos, con una media 62.45 (DE: \pm 7.23 ). La puntuación de acuerdo con las categorías de la actitud: positivas hacia la lactancia artificial (17-48 puntos), actitud neutral (49-69 puntos) o positiva hacia la lactancia materna (70-85 puntos) ${ }^{11}$ se observan en el Cuadro 2.

Intención de lactancia materna

La mayoría de las participantes ya había decidido el tipo de alimentación a ofrecer al neonato (sólo $1.8 \%$ de ellas no habían decidido aún). De la totalidad de las participantes $16 \%$ respondieron que poseen la intención de ofrecer lactancia materna exclusiva (ver Cuadro 1).

\section{Análisis de reactivos/ fiabilidad}

A través del alfa de Cronbach se obtuvo una consistencia interna de las puntuaciones del instrumento IIFAS-e, en la población de mujeres primigestas, en Monterrey, entre 0.65 y 0.68 en elementos tipificados. Sólo 8 ítems obtuvieron un valor $>$ de 0.30 correlación ítem- total (Cuadro 3).

\section{Validez de constructo}

Se determinó la prueba de adecuación muestral (KMO: 0.744) y la prueba de esfericidad de Bartlett que mostró significancia estadística ( $c^{2}$ 942.03; $\mathrm{p}=0.000$ ) que indicaron la adecuación de los datos para el análisis. Se realizó análisis factorial exploratorio con el método de extracción de factorización de ejes principales, con método de rotación Oblimin y se identificaron tres factores con el gráfico de sedimentación, con autovalores mayores a 1 que explicaron el $39.34 \%$ de la varianza total; asimismo, tomando en cuenta la teoría y la solución de números de factores encontrados ${ }^{28}$ (Cuadros 4 y 5 ).

\section{Validez predictiva}

Se realizó correlación de Spearman con actitud hacia alimentación infantil (IIFAS- español México) y la intención del tipo de lactancia elegida, con una $r=0.27 \mathrm{p}=0.000$.

\section{DISCUSIÓN}

Las características psicométricas del instrumento IIFAS español versión México, se realizaron en el presente estudio. La consistencia interna ( $\alpha$ de Cronbach 0.65) y con elementos tipificados de 0.68 , se obtuvo un resultado menor al instrumento original (0.85), ${ }^{11}$ aunque el $\alpha$ de Cronbach obtenido en nuestro estudio es muy parecido a los elaborados en diferentes idiomas como: el chino $0.62,{ }^{29} \mathrm{el} \mathrm{rumano}$ (entre 0.50 y 0.63$)^{16} \mathrm{o}$ el idioma chino en dos contextos diferentes: China y Australia con resultados desde 0.55-0.69, ${ }^{30} \mathrm{y}$ aplicado en Taiwan con $0.74 .{ }^{17}$ Durante la fase 
Cuadro1. Características sociodemográficas y clínicas de las participantes

\begin{tabular}{|c|c|c|c|}
\hline Característica & & $\mathbf{N}$ & $(\%)$ \\
\hline Edad & & Media: 25.6 & (DE: 5.9) \\
\hline \multirow[t]{3}{*}{ Localidad } & Monterrey y área metropolitana & 366 & (95) \\
\hline & Otros municipios de Nuevo León & 15 & (4) \\
\hline & Fuera de Nuevo León & 4 & $(1)$ \\
\hline \multirow[t]{6}{*}{ Educación } & Primaria & 11 & (3) \\
\hline & Secundaria & 123 & $(32)$ \\
\hline & Preparatoria & 154 & $(40)$ \\
\hline & Técnica & 4 & $(1)$ \\
\hline & Licenciatura & 92 & $(24)$ \\
\hline & Postgrado & 1 & $(0)$ \\
\hline \multirow[t]{4}{*}{ Situación laboral } & Ama de casa & 139 & (36) \\
\hline & Estudiante & 11 & $(3)$ \\
\hline & Empleada & 231 & $(60)$ \\
\hline & Empresaria & 4 & $(1)$ \\
\hline \multirow[t]{5}{*}{ Estado civil } & Casada & 246 & (64) \\
\hline & Soltera & 42 & $(11)$ \\
\hline & Divorciada & 1 & $(0)$ \\
\hline & Unión libre & 95 & $(25)$ \\
\hline & Separada & 1 & $(0)$ \\
\hline \multirow[t]{6}{*}{ Nivel socioeconómico } & Pobreza extrema & 0 & $(0)$ \\
\hline & Pobre & 23 & (6) \\
\hline & Media baja & 135 & (35) \\
\hline & Media & 88 & (23) \\
\hline & Media alta & 104 & $(27)$ \\
\hline & Rica & 35 & (9) \\
\hline \multirow[t]{3}{*}{ Trimestre de gestación } & Primero & 31 & $(8)$ \\
\hline & Segundo & 77 & $(20)$ \\
\hline & Tercero & 277 & $(72)$ \\
\hline Enfermedad presente & Sí & 127 & (33) \\
\hline \multirow[t]{5}{*}{ Tipo de enfermedad } & Diabetes tipo 1 & 33 & (9) \\
\hline & Diabetes tipo 2 & 6 & (1) \\
\hline & Diabetes gestacional & 38 & (10) \\
\hline & Hipertensión arterial & 9 & $(2)$ \\
\hline & Otras & 41 & (11) \\
\hline Pláticas prenatales & Sí & 250 & $(65)$ \\
\hline \multirow[t]{4}{*}{ Frecuencia de pláticas prenatales } & 1-3 pláticas & 200 & $(52)$ \\
\hline & $4-6$ & 42 & (11) \\
\hline & $7-9$ & 5 & $(1)$ \\
\hline & $10-12$ & 3 & (1) \\
\hline \multirow[t]{4}{*}{ Intención de lactancia } & Lactancia artificial exclusiva & & \\
\hline & Lactancia mixta & $\begin{array}{c}47 \\
273\end{array}$ & $\begin{array}{l}12 \\
71\end{array}$ \\
\hline & Lactancia materna exclusiva & 58 & 15 \\
\hline & No decisión & 7 & 2 \\
\hline $\begin{array}{l}\text { Nota: se redondearon los } \\
\text { porcentajes }\end{array}$ & & $\begin{array}{c}\text { Monterrey, N.L., } \\
\text { México. Adaptación de } \\
\text { escala de alimentación } \\
\text { al idioma }\end{array}$ & $\begin{array}{l}\text { 2014. Encuesta de } \\
\text { actitud hacia español } \\
\text { en México }\end{array}$ \\
\hline
\end{tabular}


Cuadro 2. Categorías de actitudes hacia la alimentación infantil (IIFAS-e-México) en mujeres embarazadas

\begin{tabular}{lcc}
\hline Actitudes hacia la lactancia & N & $(\%)$ \\
Actitud positiva hacia la lactancia artificial & 6 & $(1.5)$ \\
Actitud neutral & 316 & $(82.2)$ \\
Actitud positiva hacia la lactancia materna & 63 & $(16.3)$
\end{tabular}

IIFAS: Escala de Actitudes hacia la Alimentación Infantil de lowa.

Monterrey, N.L., México. 2014. Encuesta de Adaptación de escala de actitud hacia alimentación al idioma español en México. de redacción del presente manuscrito se publicó el estudio de validación al español en Colombia, con $\alpha$ de Cronbach 0.70.31

Se conservaron todos los ítems, ya que la eliminación de los ítems 1, 5 y 17 no aumentaba considerablemente el $\alpha$ de Cronbach total. La interpretación del $\alpha$ de Cronbach total de la escala de 0.65 , o de 0.68 de elementos tipificados, de acuerdo al límite "clásico" de 0.7 puede

Cuadro 3. Análisis de ítems de IIFAS versión español en México

\begin{tabular}{|c|c|c|c|}
\hline Ítems & Media (DE) & $\begin{array}{l}\text { Correlación elemento- } \\
\text { total corregida }\end{array}$ & $\begin{array}{l}\text { Alfa de Cronbach si se } \\
\text { elimina el elemento }\end{array}$ \\
\hline $\begin{array}{l}\text { 1. Los beneficios nutricionales de la leche materna (seno } \\
\text { materno) duran solo hasta que se le deja de dar }\end{array}$ & $3.04(1.26)$ & 0.157 & 0.657 \\
\hline $\begin{array}{l}\text { 2. Alimentar con leche de fórmula (de bote) es más ade- } \\
\text { cuado que dar pecho }\end{array}$ & $4.26(0.93)$ & 0.388 & 0.628 \\
\hline $\begin{array}{l}\text { 3. La lactancia materna (dar pecho) aumenta la unión } \\
\text { madre-hijo }\end{array}$ & $4.23(1.00)$ & 0.346 & 0.631 \\
\hline 4. La lactancia materna es deficiente en hierro & $3.45(1.33)$ & 0.347 & 0.628 \\
\hline $\begin{array}{l}\text { 5. Los bebés alimentados con fórmula son más propensos } \\
\text { a ser sobrealimentados que los bebés alimentados con } \\
\text { leche materna }\end{array}$ & $3.08(1.08)$ & 0.130 & 0.658 \\
\hline $\begin{array}{l}\text { 6. La alimentación con leche de bote es la mejor elección } \\
\text { si la madre planea trabajar fuera del hogar }\end{array}$ & $2.89(1.15)$ & 0.240 & 0.644 \\
\hline $\begin{array}{l}\text { 7. Madres que alimentan con leche de fórmula a sus bebés } \\
\text { pierden una de las más grandes alegrías de la maternidad }\end{array}$ & $3.34(1.20)$ & 0.252 & 0.643 \\
\hline $\begin{array}{l}\text { 8. Las madres no deberían dar pecho en lugares públicos } \\
\text { como restaurantes }\end{array}$ & $3.42(1.23)$ & 0.243 & 0.644 \\
\hline $\begin{array}{l}\text { 9. Los bebés alimentados con leche materna son más sanos } \\
\text { que aquellos alimentados por fórmula }\end{array}$ & $4.26(1.01)$ & 0.368 & 0.629 \\
\hline $\begin{array}{l}\text { 10. Los bebés alimentados con leche materna son más pro- } \\
\text { pensos a ser sobrealimentados que los bebés alimentados } \\
\text { con leche de bote }\end{array}$ & $3.37(1.13)$ & 0.207 & 0.649 \\
\hline $\begin{array}{l}\text { 11. Los papás se sienten excluidos si la madre da pecho } \\
\text { a su bebé }\end{array}$ & $4.09(0.94)$ & 0.264 & 0.642 \\
\hline 12. La leche materna es el alimento ideal para los bebés & $4.67(0.67)$ & 0.355 & 0.637 \\
\hline $\begin{array}{l}\text { 13. La leche materna es mucho más fácil de digerir que } \\
\text { la leche de fórmula }\end{array}$ & $4.15(0.91)$ & 0.339 & 0.634 \\
\hline $\begin{array}{l}\text { 14. La leche de fórmula es igual de saludable para un bebé } \\
\text { que la leche de pecho }\end{array}$ & $3.53(1.04)$ & 0.335 & 0.632 \\
\hline 15. La leche materna es más adecuada que la leche de fórmula & $4.38(0.88)$ & 0.355 & 0.632 \\
\hline 16. La leche materna es más barata que la leche de fórmula & $4.22(1.01)$ & 0.248 & 0.643 \\
\hline $\begin{array}{l}\text { 17. La mujer que ocasionalmente consume alcohol no } \\
\text { debería dar pecho a su bebé }\end{array}$ & $2.08(1.37)$ & 0.006 & 0.683 \\
\hline
\end{tabular}

IIFAS: Escala de Actitudes hacia la Alimentación Infantil de lowa.

Monterrey, N.L., México. 2014. Encuesta de Adaptación de escala de actitud hacia alimentación al idioma español en México. 
Cuadro 4. Análisis factorial exploratorio y consistencia interna de factores de versión en español México de IIFAS

\begin{tabular}{lcccc}
\hline $\begin{array}{l}\text { Factores de IIFAS-español } \\
\text { México }\end{array}$ & \multicolumn{2}{c}{$\begin{array}{c}\text { Suma de las saturaciones al cuadrado de la rotación } \\
\text { Autovalores (eigenvalues) }\end{array}$} & $\begin{array}{c}\text { Alfa de Cronbach de cada } \\
\text { factor }\end{array}$ \\
\hline 1 & 3.094 & 18.197 & 18.197 & 0.71 \\
2 & 2.222 & 13.073 & 31.271 & 0.63 \\
3 & 1.373 & 8.078 & 39.348 & 0.49 \\
\hline
\end{tabular}

IIFAS: Escala de Actitudes hacia la Alimentación Infantil de lowa.

Monterrey. N.L. México. 2014. Encuesta de Adaptación de escala de actitud hacia alimentación al idioma español en México.

Cuadro 5. Factores extraídos de Análisis Factorial Exploratorio de IIFAS-español México

\begin{tabular}{|c|c|c|c|}
\hline & \multicolumn{3}{|c|}{ Factores } \\
\hline & 1 & 2 & 3 \\
\hline & $\begin{array}{l}\text { Juicio a favor } \\
\text { LM }^{\mathrm{a}}\end{array}$ & $\begin{array}{l}\text { Juicio a favor de } \\
\text { fórmulas lácteas }\end{array}$ & $\begin{array}{l}\text { Juicios con componente } \\
\text { psicosocial y de salud }\end{array}$ \\
\hline Ítem 12 LMa: alimento ideal & .714 & & \\
\hline Ítem 15 LM: más conveniente & .651 & & \\
\hline Ítem 13 LM: mejor digerible & .586 & & \\
\hline Item 9 LM: más sana & .543 & & \\
\hline Ítem 16 LM: menos costosa & .477 & & \\
\hline Ítem 4 LM: falta hierro & & .594 & \\
\hline Ítem 11: exclusión paterna si madre da LM & & .549 & \\
\hline Ítem 2 Fórmula láctea: más adecuada & & .529 & \\
\hline Ítem 10 LM: sobrealimentación & & .444 & \\
\hline Ítem 1 beneficios nutricionales de LM & & .408 & \\
\hline Ítem 6 Formula láctea: elección si madre trabaja & & .396 & \\
\hline Ítem 14 Fórmula láctea: equivalente a LM & & .368 & \\
\hline Ítem 8 No ofrecer LM en lugares públicos & & .268 & \\
\hline Ítem 7 Formula láctea: se pierde alegría de maternidad & & & .580 \\
\hline ítem 3 LM: incrementa vinculo madre-hijo & & & .454 \\
\hline Ítem 5 Fórmula láctea: sobrealimentación & & & .400 \\
\hline Ítem 17 si tomas alcohol no dar LM & & & .174 \\
\hline
\end{tabular}

IIFAS: Escala de Actitudes hacia la Alimentación Infantil de lowa.

LM: leche materna. Método de extracción: factorización de ejes principales.

Método de rotación: Oblimin con normalización Kaiser.

considerarse baja la consistencia interna; ${ }^{20} \sin$ embargo, hay autores que mencionan que una $\alpha$ de Cronbach por encima de 0.6 se considera eficaz para juzgar una escala, ${ }^{32}$ o como lo mínimo recomendado para escalas usadas en investigación. ${ }^{27,33}$

También hay que considerar que el resultado obtenido del $\alpha$ de Cronbach, fue menor que la escala original por utilizar menos tamaño muestral ${ }^{11}$ y porque las puntuaciones obtenidas en la población fueron muy homogéneas. ${ }^{34}$

La validez de contenido de la escala IIFAS sólo se ha realizado con análisis factorial en el idioma chino, ${ }^{29}$ en estudios previos se ha realizado validez predictiva y comentando los ítems favorecedores a lactancia materna. ${ }^{11,15,17}$ 
A comparación del análisis factorial realizado por los autores en la versión china con cuatro factores, en el análisis factorial exploratorio del presente estudio, se extrajeron tres factores, con base en un criterio menos restrictivo, ${ }^{35}$ sólo el ítem 17 presentó una saturación menor de 0.25.

Los tres factores extraídos (Cuadro 5) se denominaron como juicio: 1) a favor de lactancia materna (en adelante LM); 2) a favor de fórmulas lácteas, con componente psicosocial y de salud. No se determinó la presencia de una equivalencia totalitaria de ítems al comparar los ítems de cada factor extraído, entre el análisis exploratorio de la versión china y el IIFAS-adaptado mexicano. ${ }^{29}$ La escala original no reporta análisis factorial exploratorio. ${ }^{11}$

La validez predictiva de la escala IIFAS adaptada al español en México se realizó con la prueba de correlación, determinando una correlación leve y positiva entre la actitud a la alimentación y la intención de lactancia $(r=0.27, p=0.000){ }^{36}$ La escala original reportó una correlación moderada y positiva $(r=0.35) .11,36$

A excepción de lo reportado en Colombia, donde no encontraron diferencias entre actitud y tipo de alimentación. ${ }^{31}$

\section{CONCLUSIONES}

La escala IIFAS-español México se considera válida y confiable en la población mexicana estudiada y sugerimos que se realicen más estudios con poblaciones heterogéneas. Además, hacer un Ilamado a las autoridades sanitarias de todos los niveles en la promoción de la lactancia, ya que se evidenció una actitud positiva con una intención de lactancia exclusiva solamente en bajos porcentajes.

\section{REFERENCIAS}

1. Delormier T, Frohlich KL, Potvin L. Food and eating as social practice--understanding eating patterns as social phenomena and implications for public health. Sociol Health IIIn. 2009;31(2):215-28. doi: 10.1111/j.14679566.2008.01128.x. PubMed PMID: 19220802.

2. Barker M, Swift JA. The application of psychological theory to nutrition behaviour change. Proc Nutr Soc. 2009;68(2):205-9. doi: 10.1017/S0029665109001177. PubMed PMID: 19243667.

3. McLeod ER, Campbell KJ, Hesketh KD. Nutrition knowledge: a mediator between socioeconomic position and diet quality in Australian first-time mothers. J Am Diet Assoc. 2011;111(5):696-704. doi: 10.1016/j.jada.2011.02.011. PubMed PMID: 21515115.

4. Gutiérrez JP, Rivera-Dommarco J, Shamah-Levy T, Villalpando-Hernández S, Franco A, Cuevas-Nasu L, et al. Encuesta Nacional de Salud y Nutrición 2012. Resultados Nacionales. Cuernavaca, México: Instituto Nacional de Salud Pública; 2012. 200 p. Spanish.

5. Madoka I, Collin WB, Yoko K, Mikio O. Japanese mothers' breastfeeding knowledge and attitudes assessed by the lowa Infant Feeding Attitudes Scale. Asia Pac J ClinNutr. 2013;22(2):261-265. doi: 10.6133/apjen.2013.22.2.08. PubMed PMID: 23635371.

6. Kelley MA, Kviz FJ, Richman JA, Kim JH, Short C. Development of a scale to measure gender-role attitudes toward breast-feeding among primiparas. Women Health.1993;20(1):47-68. doi: 10.1300/J013v20n01_04. PubMed PMID:8493799

7. Leff EW, Jefferis SC, Gagne MP. The development of the maternal breastfeeding evaluation scale.J Hum Lact.1994;10(2):105-111. doi: 10.1177/089033449401000217

8. Dennis CL, Faux S. Development and psychometric testing of the breastfeeding self-efficacy scale. Res Nurs Health.1999;22:399-409. doi: 10. 9.1002/(SICI)1098240X(199910)22:5<399 :AID-NUR6>3.0.CO;2-4 . PubMed PMID:10520192

9. Cleveland AP, McCrone S. Development of the breastfeeding personal efficacy beliefs inventory: a measure of women's confidence about breastfeeding. J N Meas. 2005;13(2):115-127. doi: 10.1891/jnum.2005.13.2.115. PubMed PMID:16401042

10. Janke JR. Prediction of breast-feeding attrition: instrument development. Appl Nurs Res.1992;5(1):48-53.

11. De La Mora A, Russell DW, Dungy Cl, Losch M, Dusdieker L. The lowa Infant Feeding Attitude Scale: Analysis of reliability and validity. Iowa State University. J AppISoc Psychol.1999;29(11):2362-2380.

12. Jessri M, Farmer AP, Maximova K, Willows ND, Bell RC and APrON Study Team. Predictors of exclusive breastfeeding: observations from the Alberta pregnancy outcomes and nutrition (APrON) study. BMC Pediatr. 2013;13:77. doi:10.1186/1471-2431-13-77. PubMed PMID: 23679578

13. Scott JA, Binns CW, Graham KI, Oddy WH. Temporal changes in determinants of breastfeeding initiation. Birth. 
2006;33(1):37-45. doi: 10.1111/j.0730-7659.2006.00072.x PubMed PMID: 16499530

14. Dungy $\mathrm{Cl}$, Mclnnes RJ, Tappin DM, Wallis AB, Oprescu F. Infant feeding attitudes and knowledge among socioeconomically disadvantaged women in Glasgow. Matern Child Health J. 2008;12:313-322. PubMed PMID: 17690964

15. Sittlington J, Stewart-Knox B, Wright M, Bradbury I, Scott JA. Infant-feeding attitudes of expectant mothers in Northern Ireland. Health Educ Res. 2007;22:561-570. doi: 10.1093/ her/cyl113. PubMed PMID:17041021

16. Wallis AB, Brinzaniuc A, Chereches R, Oprescu F, Sirlincan $E$. Reliability and validity of the Romanian version of a scale to measure infant feeding attitudes and knowledge. Acta Paediatr. 2008;97:1194-1199. doi: 10.1111/j.16512227.2008.00914.x. PubMed PMID:18627591

17. Ho YJ, McGrath JM. A Chinese version of lowa Infant Feeding Attitude Scale: Reliability and validity assessment. Int J Nurs Stud. 2011; 48:475-478.doi: 10.5172/conu.2013.44.1.11. PubMed PMID: 23721383.

18. Nanishi K, Jimba M. Reliability and Validity of the Japanese Version of the lowa Infant Feeding Attitude Scale: A Longitudinal Study. J Hum Lact. 2014; 30(3):346-352. PubMed PMID: 24828780.

19. Muñiz J, Elousa P, Hambleton RK. Directrices para la traducción y adaptación de los tests: segunda edición. Psicothema. 2013; 25(2):151-157. doi: 10.7334/psicothema2013.24

20. Nunnally JC. Psychometric Theory. New York: McGraw-Hill; 1978. 701p.

21. Thorndike RL. Applied Psychometrics, Boston: HoughtonMifflin; 1982. 390p.

22. Carretero-Dios H, Pérez C. Standards for the development and review of instrumental studies: Consideration about test selection in psychology research. Int J ClinHealthPsychol. 2007; 7:863-882.

23. Carvajal A, Centeno C, Watson R, Martínez M, Sanz- Rubiales $A$. ¿Cómo validar un instrumento de medida de la salud?. An Sist Sanit Navar. 2011;(34) 1. doi.org/10.4321/ S1137-66272011000100007

24. INEGI. Marco conceptual del Censo de Población y Vivienda 2010. México: INEGI; 2011. 189p.

25. López H. Presentación de conferencia de Nuevo Índice Socioeconómico Asociación Mexicana de Agencias de Investigación de Mercado y opinión Pública. AMAI 2008 CONGRESO AMAI 2008; Sept 2008 [power point]. AMAI
2008. Disponible en: www.amai.org/congreso/2008/ memorias/ponencias/lopezromo.

26. Donath SM, Amir LH, ALSPAC Study Team. Relationship between prenatal infant feeding intention and initiation and duration of breastfeeding: a cohort study. Acta Paediatr. 2003;92(3):352-6. doi: 10.1111/j.1651-2227.2003. tb00558.x PubMed PMID:12725552

27. Barrio-Cantalejo IM, Simón-Lorda P, Melguizo M, Escalona I, Marijuán MI, Hernando P. Validación de la Escala INFLESZ para evaluar la legibilidad de los textos dirigidos a pacientes. An Sist Sanit Navar. 2008;(31)2.

28. Lloret-Segura S, Ferreres-Traver A, Hernández-Baeza A, Tomás-Marco I. El análisis factorial exploratorio de los ítems: una guía práctica, revisada y actualizada. Anal. Psicol. 2014;30(3):1151-1169. http://dx.doi.org/10.6018/ analesps.30.3.199361

29. Dai HX, Guan XD ,Li XM, You LM, Lau Y. Psychometric properties of a mainland Chinese version of the lowa Infant Feeding Attitude Scale among postpartum women in China. Contemp Nurse. 2013;44(1):11-20. PubMed PMID: 23721383.

30. Chen S, Binns CW, Liu Y, Maycock B, Zhao Y, Tang L. Attitudes towards breastfeeding - the lowa Infant Feeding Attitude Scale in Chinese mothers living in China and Australia. Asia Pac J Clin Nutr. 2013;22(2):266-269. PubMed PMID: 23635372.

31. Jácome Á, Jiménez R. Validación de la lowa Infant Feeding Attitude Scale. Pediatr. 2014;47(4): 77-82.

32. Flynn B, Schroeder R, Sakakibara S. A framework for quality management research and an associated measurement instrument. Journal of Operations Management. 1994;11:339-366. http://dx.doi.org/10.1016/S02726963(97)90004-8

33. Hair JF, Black WC, Babin BJ, Anderson RE. Multivariate data analysis (7th ed.). Upper saddle River, New Jersey: Pearson Education International; 2010. 816p

34. Campo-Arias A, Oviedo HC. Propiedades Psicométricas de una Escala: la Consistencia Interna. Rev SaludPública. 2008;10 (5):831-839.

35. Floy FJ, Widaman KF. Factor analysis in the development and refinement of clinical assessment instrument. Psychol Asses. 1995;7:286-299.

36. Cohen, J. Statistical power analysis for the behavioral sciences (2nd ed.). New Jersey: Lawrence Erlbaum; 1988. 567p. 